\title{
Highlights from Studies Presented at the American Heart Association Scientific Session 2020: Navigating New Roads in Prevention
}

\author{
Aliza Hussain ${ }^{1} \cdot$ Mahmoud Al Rifai $^{1} \cdot$ Dhruv Mahtta $^{1} \cdot$ Jing Liu $^{1} \cdot$ Vardhmaan Jain $^{2} \cdot$ Salim S. Virani $^{3,1}$ (D) \\ Accepted: 17 December 2020 / Published online: 3 January 2021 \\ (C) This is a U.S. government work and not under copyright protection in the U.S.; foreign copyright protection may apply 2021
}

\begin{abstract}
Purpose of the Review This review highlights late-breaking science presented at the American Heart Association Scientific Session 2020 that demonstrated advancements in preventative cardiology and introduced novel treatment approaches for the management of chronic kidney disease, type 2 diabetes, and/or heart failure.

Recent Findings The studies reviewed include clinical trials that assessed the use of omecamtiv in the treatment of heart failure with reduced heart failure (GALACTIC-HF); effects of sotagliflozin in patients with diabetes and recent heart failure exacerbation; cardiovascular outcomes with the use of omega-3 carboxylic acids in patients with high vascular risk and atherogenic dyslipidemia (STRENGTH) and omega-3 fatty acids in elderly patients with recent myocardial infarction (OMEMI); efficacy and safety of evinacumab in patients with refractory hypercholesterolemia; and the use of coronary computed tomography angiography for the assessment of suspected acute coronary syndrome. In addition, we review the results of the International Polycaps Study (TIPS-3) on the use of a polypill for the primary prevention of cardiovascular disease in intermediate-risk people. Finally, we discuss the SAMSON trial-a three-arm-N-of-1 trial-to identify the root cause of the symptoms contributing to patient nonadherence to statin therapy.

Summary The studies presented at the American Heart Association Scientific Session 2020 represent remarkable contributions in the field of cardiovascular disease and prevention.
\end{abstract}

Keywords Cardiovascular prevention - Statins · Polypill $\cdot$ Coronary computed tomography angiography $\cdot$ Sodium-glucose co-transporter inhibitors · Omega-3 fatty acids

\section{Introduction}

The American Heart Association (AHA) Scientific Session 2020 was held virtually this year due to the ongoing coronavirus disease 2019 (COVID-19) pandemic. It featured multiple late-breaking science and notable studies in the field of

This article is part of the Topical Collection on Reviews and New Research Implications

Salim S. Virani

virani@bcm.edu

Aliza Hussain

alizah@bcm.edu

Mahmoud Al Rifai

mahmoud.alrifai@bcm.edu

Dhruv Mahtta

dhruv.mahtta@bcm.edu

Jing Liu

jing.liu@bcm.edu heart failure, diabetes, chronic kidney disease, and cardiovascular prevention. In this review, we summarize key findings from some of the major late-breaking clinical trial and additional select studies relevant to cardiovascular disease (CVD) prevention that were presented at the AHA Scientific Session 2020 .

Vardhmaan Jain

jainv2@ccf.org

1 Section of Cardiology Department of Medicine, Baylor College of Medicine, Houston, TX, USA

2 Department of Internal Medicine, Cleveland Clinic Foundation, Cleveland, OH, USA

3 Section of Cardiology Health Services Research and Development, Michael E. DeBakey Veterans Affairs Medical Center, 2002 Holcombe Boulevard, Houston, TX 77030, USA 
The studies include cardiovascular outcome trials evaluating the effects of omecamtiv [1•] and sotagliflozin [2••] on cardiovascular outcomes in patients with heart failure. We further highlight data from the STRENGTH (Long-Term Outcome Study to Assess Statin Residual Risk Reduction With Epanova in High Cardiovascular Risk Patients with Hypertriglyceridemia) trial assessing the effects of on omega-3 carboxylic acids (Epanova) cardiovascular outcomes in patients with high vascular risk and atherogenic dyslipidemia [3••]; the OMEMI (Omega-3 Fatty Acids in Elderly Patients With Acute Myocardial Infarction) trial assessing effects of omega-3 fatty acids in elderly patients after myocardial infarction [4]; a phase 2 clinical trial studying efficacy and safety of evinacumab in patients with refractory hypercholesterolemia [5]; and RAPID-CTCA (Rapid Assessment of Potential Ischaemic Heart Disease with CTCA) trial studying the use of coronary computed tomography angiography for assessment of suspected acute coronary syndrome [6•]. In addition, we summarize the results of the International Polycaps Study (TIPS-3) on the use of a polypill for primary prevention of cardiovascular disease in intermediate-risk people [7], and, finally, discuss the SAMSON (Self-Assessment Method for Statin Side-Effects or Nocebo) trial - a three-armN-of-1 trial - to identify the root cause of the symptoms contributing to patient nonadherence to statin therapy [8•]. As in prior publications, we aim to provide a summary for each of select major studies and their clinical implications [9-12].

\section{Omecamtiv Mecarbil in Chronic Heart Failure with Reduced Ejection Fraction}

\section{Study Overview}

Omecamtiv mecarbil is a cardiac myosin activator. The GALACTIC-HF (Global Approach to Lowering Adverse Cardiac Outcomes Through Improving Contractility in Heart Failure) trial was a multi-center, phase 3, randomized, placebo-controlled trial which assessed the safety and efficacy of omecamtiv mecarbil in 8256 patients with heart failure with reduced ejection fraction (HFrEF) with New York Heart Association (NYHA) functional class II-IV and LVEF $\leq$ $35 \%[1 \bullet]$.

At a median follow-up of 21.8 months, the primary outcome of cardiovascular death or heart failure (HF) event occurred in $37.0 \%$ vs. $39.1 \%$ (hazard ratio [HR]: 0.92 , $95 \%$ confidence interval $(\mathrm{CI}): 0.86-0.99 ; P=0.03)$ of patients receiving omecamtiv mecarbil and placebo, respectively. The positive effect of omecamtiv mecarbil was consistent across all pre-specific subgroups except for the subgroup of patients with LVEF $>$ median $(>28 \%)$, who did not appear to derive benefit as compared to those with LVEF $\leq$ $28 \%$. Secondary endpoints including cardiovascular death, first HF hospitalization, and any-cause mortality were similar between the two treatment arms. Although change in Kansas City Cardiomyopathy Questionnaire (KCCQ)-12 score from baseline to 24 weeks was higher among patients receiving omecamtiv mecarbil compared to placebo $(23.7 \%$ vs. $21.1 \%$, relative risk (RR): $0.97,95 \% \mathrm{CI}: 0.85$ to 1.11$)$ ), this did not reach statistical significance. The rate of drug discontinuation and safety endpoints including myocardial ischemia and ventricular arrhythmic events were similar in both arms.

In terms of safety, rates of drug discontinuation were similar $(20.6 \%$ in omecamtiv mecarbil vs $21.9 \%$ in the placebo group). At week 24, the median change in cardiac troponin I level was $4 \mathrm{ng}$ per liter higher in the omecamtiv mecarbil group compared to placebo. However, rates of major cardiac ischemic events, ventricular arrhythmias, and stroke were similar in both arms.

\section{Clinical Implications}

Building on the results of the phase 2 COSMIC-HF (Chronic Oral Study of Myosin Activation to Increase Contractility in Heart Failure) trial [13], the GALACTIC-HF trial demonstrated a modest improvement ( $\sim 8 \%$ relative risk reduction and $2.1 \%$ absolute risk reduction) in the composite of cardiovascular death and HF events among patients with HFrEF. Improvement in individual components of the primary endpoint (cardiovascular mortality, all-cause mortality, HF hospitalization) or symptomatic improvement (based on KCCQ score), did not reach statistical significance. Despite this, the overall findings from this trial deserve clinical merit given the inclusion of a broad set of patients: those inpatient and outpatient, with moderate to severe HF symptoms (NYHA functional class III and IV), reduced median LVEF, lower systolic blood pressure, and advanced renal disease. These findings provide initial evidence for the potential use of this therapy among a subgroup of patients with advanced renal disease, advanced HF, or low systolic blood pressure who may not otherwise tolerate other classes of HF therapy.

Although patients receiving omecamtiv mecarbil had increased troponin levels, overall adverse event rates (myocardial ischemia and ventricular arrhythmias) were similar across both groups during $~ 7500$ patient-years follow-up.

\section{Effect of Sotagliflozin on Cardiovascular Events in Patients with Type 2 Diabetes and Heart Failure (SOLOIST-WHF Trial)}

\section{Study Overview}

SOLOIST-WHF was a multi-center, double-blind, randomized, placebo-controlled trial that was designed to investigate 
the safety and efficacy of sotagliflozin in reducing adverse cardiovascular events among patients with type 2 diabetes mellitus (T2DM) and recent hospitalization for decompensated HF [2]. The originally designed primary endpoint included the first occurrence of either death from cardiovascular causes or HF hospitalization. However, due to loss of funding from the sponsor amidst the COVID-19 pandemic and early termination of the trial, the primary endpoint was changed to include a composite of cardiovascular mortality, HF hospitalizations, and urgent visits for HF.

A total of 1222 patients with recent admission with HF and previous diagnosis of T2DM were enrolled and randomized in a $1: 1$ fashion to either receive sotagliflozin $400 \mathrm{mg}$ daily $(n=608)$ or placebo $(n=614)$. At a median follow-up of 9 months, the rate of primary endpoint events was 51.0 vs. 76.3 events/100 patient-years (HR: $0.67,95 \%$ CI: $0.52-0.85 ; P<0.001)$. Significance was achieved as early as 28 days during follow-up. Consistent treatment effect was observed across subgroups of patients stratified by timing of the first dose and LVEF ( $<50 \%$ vs. $\geq 50 \%)$. The rates of cardiovascular mortality were 10.6 vs. 12.5 events/100 patients-years $(p=0.36)$, while the change in KCCQ-12 score was 17.7 vs. $13.6(p<0.01)$, in favor of sotagliflozin.

Safety endpoints including diarrhea $(6.9 \%$ vs. $4.1 \%, P=$ $0.032)$ and severe hypoglycemia $(1.5 \%$ vs. $0.3 \%, P=0.037)$ were more common among patients receiving sotagliflozin while rates of acute kidney injury, genital mycotic infections, and hypotension were similar among both treatment arms.

\section{Clinical Implications}

Although the early termination of this trial compromised the ability to define reductions in individual components of the primary outcome, the overall results are highly encouraging and consistent across multiple prespecified subgroups (initiation before or shortly after discharge, baseline LVEF $<50 \%$ or $\geq 50 \%$ ).

The results of this trial are consistent with those observed with empagliflozin in the EMPEROR-Reduced trial [14] and with dapagliflozin in the DAPA-HF trial [15]. However, this study also provides reassuring evidence regarding the safety and efficacy of sotagliflozin which provides sodium-glucose cotransporter-1 (SGLT1) inhibition (delaying post-prandial gastrointestinal glucose absorption) in addition to SGLT2 inhibition (glucose elimination in urine). Moreover, this trial advances the field of SGLT2inhibition by extending the efficacy of these agents among patients hospitalized for acute decompensated HF (after clinical stabilization). The results achieved significance at a median of 28 days which also highlights the early effect of these agents and thus the importance of starting these agents before or soon after hospital discharge.
Effect of High-Dose Omega-3 Fatty Acids on Cardiovascular Events in Patients at High Cardiovascular Risk

\section{Study Overview}

The STRENGTH trial was a multi-centric, double-blinded, randomized, placebo-controlled trial of 13,078 patients spanning 22 countries, assessing the safety and efficacy of a carboxylic acid formulation of EPA and DHA (omega-3 CA) administered at $4 \mathrm{~g}$ /day compared with corn oil, in addition to background therapy in the reduction of risk for atherosclerotic CVD (ASCVD) events in statin-treated patients at high cardiovascular risk [3]. Patients enrolled had either (a) established ASCVD (secondary prevention) or (b) type 1 or type 2 diabetes (age $>$ 40 for men and age $>50$ for women) with one additional risk factor or (c) high-risk primary prevention patients (age $>60$ for women and $>50$ for men) with one additional cardiovascular risk factor. All participants had low-density lipoprotein cholesterol (LDL-C) $<100 \mathrm{mg} / \mathrm{dL}$, had been on statin therapy for at least 4 weeks, had triglyceride levels of 180 to $500 \mathrm{mg} / \mathrm{dL}$, and had high-density lipoprotein cholesterol (HDL-C) less than $42 \mathrm{mg} / \mathrm{dL}$ for men and $47 \mathrm{mg} / \mathrm{dL}$ for women. The trial was terminated prematurely when 1384 patients had experienced a primary endpoint (of a planned 1600 events to show a $15 \%$ trial drug efficiency with a power of $90 \%$ ) based on an interim analysis that indicated a low probability of clinical benefit of omega-3 CA vs the corn oil comparator.

At baseline, mean LDL-C level was $75.0 \mathrm{mg} / \mathrm{dL}$, median triglyceride level $240 \mathrm{mg} / \mathrm{dL}$, and median HDL-C level $36 \mathrm{mg} /$ $\mathrm{dL}$. The primary endpoint (composite of cardiovascular death, nonfatal myocardial infarction, nonfatal stroke, coronary revascularization, or unstable angina requiring hospitalization) occurred in 785 patients $(12.0 \%)$ treated with omega-3 CA vs $795(12.2 \%)$ treated with corn oil (HR 0.99, 95\% CI: 0.90 $1.09 ; P=0.84)$. A greater rate of gastrointestinal adverse events was observed in the omega-3 CA group (24.7\%) compared with corn oil-treated patients $(14.7 \%)$ which contributed to greater trial drug discontinuation compared with placebo. Notably, an increased rate of investigator-reported atrial fibrillation was observed in the omega-3 CA group (2.2\% vs $1.3 \%$; HR, $1.69,95 \%$ CI, 1.29-2.21; nominal $P<0.001$ ) compared with corn oil (number needed to harm, 114), similar to the trends seen in prior trials comparing purified EPA to mineral oil [16].

\section{Effects of n-3 Fatty Acid Supplements in Elderly Patients After Myocardial Infarction}

\section{Study Overview}

The OMEMI trial was a randomized, placebo, double-blinded trial that randomized 1027 elderly patients (mean age 74 years, 
$29.3 \%$ female) with recent myocardial infarction (2-8 weeks post-event) receiving background standard therapy to receive either $1.8 \mathrm{~g} \mathrm{n}-3$ fatty acids (930 mg EPA/660 mg DHA) or corn oil-based placebo [4]. Mean triglyceride levels were $111.4 \mathrm{mg} / \mathrm{dL}$. After a follow-up period of 24 months, the primary outcome of all-cause death, nonfatal myocardial infarction, unscheduled revascularization (stent or bypass surgery), stroke, or hospitalization for heart failure occurred in $21.0 \%$ of the PUFA group compared with $19.8 \%$ of the placebo group $(p=0.62)$. There was no heterogeneity in treatment effect across the subgroups of patients.

Treatment-related adverse events included an increased rate of atrial fibrillation (7.2\% in the treatment arm compared with $4.0 \%$ in the placebo arm, $P=0.06$ ) and gastrointestinal bleeding $(10.7 \%$ in the treatment arm compared with $11.0 \%$ in the placebo arm, $P=0.87)$. The trial failed to show a beneficial effect of PUFA in preventing adverse cardiovascular events in elderly patients with recent myocardial infarction.

\section{Clinical Implications}

The STRENGTH and the OMEMI trial add to an increasing body of evidence aimed at assessing the efficacy of fish oil-based therapies in primary or secondary ASCVD prevention. Importantly, both trials did not show a benefit. Omega-3 fatty acids have been shown to have a wide range of biological effects on inflammation, the composition of biological membranes, oxidative stress, and characteristics of atherosclerotic plaques [17]. Earlier trials that used lower doses of EPA failed to show a beneficial cardioprotective benefit $[18,19]$. However, the JELIS [20] and the REDUCE-IT [16] trials used a purified form of EPA and found a $19 \%$ and $25 \%$ reduction in the risk for adverse ASCVD events, respectively [21], as discussed in detail elsewhere. The JELIS trial was an open-label trial, with a less aggressive background statin therapy and a population that had higher rates of dietary fish oil consumption. The REDUCE-IT trial used mineral oil as the placebo. STRENGTH and the OMEMI trials, both of which were neutral studies that used an inert comparator-corn oil-provide important insight. Table 1 highlights some of the key differences between these four trials. It is also important to note that given the small sample size, normal triglyceride levels, and short follow-up in OMEMI, the trial was underpowered to show an impact.

Whether the results seen in STRENGTH and OMEMI were driven by the presence of a non-neutral comparator in REDUCE-IT, or whether the results were offset by using an EPA-DHA combination in STRENGTH and OMEMI, remains unanswered. Most likely, this is a combination of both of the above points as EPA may be more beneficial compared to DHA and the significant relative and absolute risk reduction seen in REDUCE-IT would be very difficult to explain by the small increase in LDL-C and the increase in hs-CRP noted in patients receiving mineral oil placebo in REDUCE-IT. Regardless, a consistent theme has been an increased reporting of atrial fibrillation in these trials, a fact that should be considered when clinicians prescribe these agents to their high-risk patients.

\section{Efficacy and Safety of Monoclonal Antibody Treatments in Refractory Hypercholesterolemia}

Patients with severe hypercholesterolemia, defined as an LDL-C of greater than $190 \mathrm{mg} / \mathrm{dL}$, have a 6 times higher risk of coronary artery disease (even without a genetic mutation), compared to those with LDL-C less than $130 \mathrm{mg} / \mathrm{dL}$ [22] due to deleterious effects from lifetime exposure to elevated LDLC levels. Despite the use of maximally tolerated statins, ezetimibe, and proprotein convertase subtilisin-kexin type 9 (PCSK9) inhibitor, some patients with severe hypercholesterolemia need additional lipid-lowering agents based on multisociety guideline specific goals/threshold.

\section{Study Overview}

Angiopoietin-like protein 3 (ANGPTL3) is a hepatic secretory protein that inhibits lipoprotein lipase and endothelial lipase. Patients with loss of function mutations in ANGPTL3 demonstrate significantly reduced levels of LDL-C, high-density lipoprotein cholesterol (HDL-C), and triglycerides [23]. Evinacumab is a fully human monoclonal antibody that neutralizes the effect of ANGPTL3. A previous phase 3 study of evinacumab in patients with homozygous familial hypercholesterolemia $(\mathrm{FH})$ showed a between-group mean difference of $49 \%$ in reduction in LDL-C compared to placebo [5]. The present phase 2 clinical trial sought to evaluate the efficacy and safety of subcutaneous (SC) and intravenous (IV) evinacumab, compared to placebo, in patients with refractory hypercholesterolemia.

The trial enrolled patients who had a diagnosis of primary hypercholesterolemia (either heterozygous $\mathrm{FH}$ or non-FH with clinical ASCVD), were receiving stable lipid-lowering therapy with statin \pm ezetimibe at the maximum tolerated dose for at least 4 weeks and PCSK9 inhibitor for at least 8 weeks, and had an LDL-C $\geq 70 \mathrm{mg} / \mathrm{dL}$ or $\geq 100 \mathrm{mg} / \mathrm{dL}$ with or without ASCVD. Participants were randomly assigned to receive either SC evinacumab $(n=160$; randomized $1: 1: 1: 1$ to $450 \mathrm{mg}$ weekly, $300 \mathrm{mg}$ weekly, $300 \mathrm{mg}$ every 2 weeks or matching placebo every week) or IV evinacumab $(n=106$ randomized 1:1:1 to $15 \mathrm{mg} / \mathrm{kg}$ every 4 weeks, $5 \mathrm{mg} / \mathrm{kg}$ every 4 weeks or placebo). 
Table 1 Comparison of the major cardiovascular outcomes clinical trials of high-dose EPA or high-dose EPA and DHA

\begin{tabular}{|c|c|c|c|c|}
\hline & JELIS [20] & REDUCE-IT [16] & STRENGTH [3] & OMEMI [4] \\
\hline Patient population & $\begin{array}{l}\text { Hypercholesterolemia, men } \\
40-75 \text { yrs. or postmenopausal } \\
\text { women up to age } 75\end{array}$ & $\begin{array}{l}\text { (1) Established ASCVD or } \\
\text { (2) DM and age } \geq 50 \text { yrs. } \\
\text { with and one additional } \\
\text { ASCVD risk factor }\end{array}$ & $\begin{array}{l}\text { (1) Established ASCVD or (2) DM and } \\
\text { age } \geq 40 \text { yrs. for men or } \geq 50 \text { yrs. women } \\
\text { with and one additional ASCVD risk } \\
\text { factors or (3) age } \geq 50 \text { yrs. for men } \\
\text { and } \geq 60 \text { yrs. for women with } \geq 1 \\
\text { additional ASCVD risk factor }\end{array}$ & $\begin{array}{l}\text { Age } 72-82 \text { yrs. } \\
\text { with MI } \\
<2-8 \text { weeks }\end{array}$ \\
\hline Study design & PROBE & RCT & $\mathrm{RCT}$ & RCT \\
\hline Location & Multi-center, Japan & Multicenter, multinational & Multicenter, multinational & $\begin{array}{l}\text { Multicenter, } \\
\text { Norway }\end{array}$ \\
\hline Baseline lipid levels & $\mathrm{TC} \geq 250 \mathrm{mg} / \mathrm{dL}$ & $\begin{array}{l}\text { TG } 150-500 \mathrm{mg} / \mathrm{dL} \\
\quad(135 \mathrm{mg} / \mathrm{dL} \text { if on statin })\end{array}$ & TG $180-500 \mathrm{mg} / \mathrm{dL}$ & No specific criteria \\
\hline Study size, $n$ & 18,645 & 8179 & 13,078 & 1027 \\
\hline Drug therapy & $1800 \mathrm{mg}$ EPA & 4000 mg EPA & 4000 mg EPA/DHA (omega-3 CA) & $\begin{array}{l}1.8 \mathrm{~g} \mathrm{n}-3 \text { PUFA } \\
(930 \mathrm{mg} \text { EPA } \\
\text { and } 660 \mathrm{mg} \\
\text { DHA) }\end{array}$ \\
\hline Placebo & None & Mineral oil & Corn oil & Corn oil \\
\hline \multicolumn{5}{|l|}{ Baseline characteristics } \\
\hline Age, years & 61 & 64 & 63 & 75 \\
\hline Female, $\%$ & 69 & 29 & 35 & $29 \%$ \\
\hline White, \% & - & $92 \%$ & $82 \%$ & $100 \%$ \\
\hline $\begin{array}{l}\text { Secondary vs. primary } \\
\text { prevention }\end{array}$ & $20 \%$ vs $80 \%$ & $71 \%$ vs $29 \%$ & $56 \%$ vs $44 \%$ & $\begin{array}{l}\text { All secondary } \\
\text { prevention }\end{array}$ \\
\hline Statin use & $97-98 \%$ & $100 \%$ & $100 \%$ & $96 \%$ \\
\hline High intensity statin & 0 & $30 \%$ & $50 \%$ & $\mathrm{n} / \mathrm{a}$ \\
\hline Ezetimibe & $\mathrm{n} / \mathrm{a}$ & $6.4 \%$ & $3.7 \%$ & $\mathrm{n} / \mathrm{a}$ \\
\hline LDL-C, mg/dL & 182 & 76 & 75 & 76 \\
\hline $\mathrm{TG}, \mathrm{mg} / \mathrm{dL}$ & 151 & 216 & 240 & 110 \\
\hline HDL-C, mg/dL & 58 & 40 & 36 & 50 \\
\hline EPA levels & $\mathrm{n} / \mathrm{a}$ & $26.1 \mu \mathrm{g} / \mathrm{mL}$ & $21 \mu \mathrm{g} / \mathrm{mL}$ & 2.9 (\%wt) \\
\hline \multicolumn{5}{|l|}{ Outcomes } \\
\hline Follow-up, years & 4.6 & 4.9 & 3.5 & 2 \\
\hline Primary endpoint & $\mathrm{SCD}, \mathrm{UA}, \mathrm{MI}$, or cor revasc & $\begin{array}{l}\mathrm{CV} \text { death, nonfatal MI, } \\
\text { nonfatal stroke, cor } \\
\text { revasc, or UA }\end{array}$ & CV death, MI, stroke, cor revasc, or UA & $\begin{array}{l}\text { Non-fatal MI, cor } \\
\text { revasc, stroke, } \\
\text { all-cause death, } \\
\text { HF } \\
\text { hospitalization }\end{array}$ \\
\hline $\begin{array}{l}\text { Outcome HR (95\% } \\
\quad \text { CI) }\end{array}$ & $2.8 \%$ vs $3.5 \%(0.81,0.69-0.95)$ & $\begin{array}{l}17.2 \% \text { vs } 22 \%(0.75 \\
0.68-0.83)\end{array}$ & $12 \%$ vs $12.2 \%(0.99,0.90-1.09)$ & $\begin{array}{l}21 \% \text { vs } 19.8 \% \\
\quad(1.07,0.82-1.40)\end{array}$ \\
\hline Notable differences & $\begin{array}{l}\text { Open label study, only low intensity } \\
\text { statin either pravastatin }(10 \mathrm{mg} / \mathrm{d}) \\
\text { or simvastatin }(5 \mathrm{mg} / \mathrm{d}) \text { used. } \\
\text { Elevated LDL-C at baseline }\end{array}$ & $\begin{array}{l}\text { Placebo event rates much } \\
\text { higher in REDUCE-IT } \\
(22 \%) \text { vs STENGTH } \\
(12 \%)\end{array}$ & $\begin{array}{l}\text { Coronary events lowered in STRENGTH but } \\
\text { at nominal } P \text { value and not adjusted for } \\
\text { hierarchical testing }\end{array}$ & $\begin{array}{l}\text { Short follow up and } \\
\text { small study size } \\
\text { compared to the } \\
\text { other three trials }\end{array}$ \\
\hline
\end{tabular}

JELIS = Japan EPA Lipid Intervention Study; REDUCE-IT = Japan EPA Lipid Intervention Study; STRENGTH = Long-Term Outcome Study to Assess Statin Residual Risk Reduction With Epanova in High Cardiovascular Risk Patients with Hypertriglyceridemia; OMEMI = omega-3 fatty acids in elderly patients with recent myocardial infarction; RCT =randomized controlled trial (double-blind, placebo-controlled); PROBE = prospective randomized open blinded endpoint; $\mathrm{TG}=$ triglycerides; $\mathrm{TC}=$ total cholesterol; LDL-C $=$ low-density lipoprotein cholesterol; HDL-C $=$ high-density lipoprotein cholesterol; $\mathrm{HR}=$ hazard ratio $\mathrm{CI}=$ confidence interval; $\mathrm{CV}=$ cardiovascular; $\mathrm{ASCVD}=$ atherosclerotic cardiovascular disease; $\mathrm{HF}=$ heart failure; $\mathrm{MI}=$ myocardial infarction; $\mathrm{DM}=$ diabetes mellitus; yrs. = years; EPA = eicosapentaenoic acid; DHA = docosahexaenoic acid; $\mathrm{n}-3$ PUFA = omega-3 polyunsaturated fatty acids; omega-3 $\mathrm{CA}=$ omega-3 carboxylic acid; $\mathrm{SCD}=$ sudden cardiac death; $\mathrm{UA}=$ unstable angina; cor revasc = coronary revascularization

The mean age of the study population was 54 , with $62 \%$ and $56 \%$ of females in the SC and IV arms. In total, $28 \%$ did not have heterozygous $\mathrm{FH}$ in the $\mathrm{SC}$ and $19 \%$ in the IV arm. Baseline LDL-C levels were $150 \mathrm{mg} / \mathrm{dL}$ and $145 \mathrm{mg} / \mathrm{dL}$ in the
SC and IV groups, respectively. Baseline use of any statin was $61 \%$, high-intensity statin $44 \%$, ezetimibe $30 \%$, and PCSK9 inhibitor $100 \%$ in the SC group. A similar trend was seen in the IV group. 
After 16 weeks, the primary effectiveness endpoint, i.e., mean percent change in LDL-C from baseline with SC evinacumab $450 \mathrm{mg}$ vs. SC evinacumab $300 \mathrm{mg}$ every week vs. SC evinacumab $300 \mathrm{mg}$ every 2 weeks vs. placebo were $47.2 \%$ vs. $-44.0 \%$ vs. $-29.7 \%$ vs. $+8.8 \%$, respectively. Similarly, in the IV arm, the mean percent change in LDL-C from baseline for IV evinacumab $15 \mathrm{mg} / \mathrm{kg}$ vs. evinacumab $5 \mathrm{mg} / \mathrm{kg}$ vs. placebo were $-49.9 \%$ vs. $-23.5 \%$ vs. $+0.6 \%$, respectively. Dose-dependent reductions of up to $31.4 \%$, $45.5 \%$, and $61.5 \%$ were also seen in HDL-C, apolipoprotein $\mathrm{B}$, and triglycerides, respectively.

In terms of safety, most common adverse events (vs placebo) included urinary tract infection (11\% vs. $8 \%$ ), injectionsite erythema (6\% vs. $3 \%$ ), arthralgia ( $5 \%$ vs. $3 \%$ ), and myalgia (5\% vs. $0 \%)$ in the $\mathrm{SC}$ arm and abdominal pain (6\% vs. $0 \%$ ), dizziness (7\% vs. $0 \%)$, nausea $(7 \%$ vs. $0 \%)$, and nasopharyngitis ( $12 \%$ vs. $6 \%$ ) for the IV arm.

\section{Clinical Implications}

This clinical trial demonstrates that evinacumab significantly reduced LDL-C, compared to placebo, among patients with refractory hypercholesterolemia (baseline LDL-C: $\sim 150 \mathrm{mg} /$ dl) already on conventional lipid-lowering therapies with statins, ezetimibe, and PCSK9 inhibitors. The reductions in LDL-C, HDL-C, and triglycerides were dose-dependent, with higher doses of either SC or IV evinacumab demonstrating a greater reduction in lipid levels, and reductions were observed within 2 weeks and sustained at 16 weeks.

The results from this evinacumab study, while promising for the treatment of refractory hypercholesterolemia, should be viewed with caution as more data is needed to ascertain its long-term efficacy, safety, and tolerability. Moreover, although the reduction in LDL-C would be associated with net clinical benefit, the effect of a significant reduction in HDL-C on clinical outcomes is not well studied. As this trial was small and underpowered to assess clinical outcomes, future large long-term clinical outcome trials in different patient populations are warranted to assess the clinical efficacy of this novel lipid-lowering therapy. Nevertheless, this trial adds to the growing body of evidence on the use of monoclonal antibodies and RNA interference (RNAi) technologies to modulate lipid levels and improve ASCVD outcomes [24].

\section{Rapid Assessment of Potential Ischaemic Heart Disease with CTCA}

The RAPID CTCA trial was a pragmatic randomized clinical trial designed to investigate the effect of early coronary computed tomography angiography (CCTA) in patients who present to the emergency room with suspected or confirmed acute coronary syndrome (ACS). The primary outcome was allcause death or non-fatal type 1 (spontaneous) or type $4 \mathrm{~b}$ (stent thrombosis) myocardial infarction (MI) at 1 year.

\section{Study Overview}

The study included 1748 patients with a mean age of 62 years including $36 \%$ women and $18 \%$ with diabetes. Patients were included if they had a prior history of coronary heart disease, troponin above the 99th percentile, or an abnormal electrocardiogram. The GRACE score upon presentation to the emergency department was $<109$ in $44 \%$ of patients, $109-140$ in $32 \%$, and $>140$ in $23 \%$. Patients in the CCTA arm were found to have normal coronary arteries in $23 \%$ of cases, nonobstructive disease in $29 \%$, and obstructive disease in $47 \%$.

The primary outcome occurred in 5.8\% of the CCTA group compared with $6.1 \%$ of the usual care group $(P=0.65)$ with no significant differences among prespecified subgroups. The use of invasive coronary angiography was significantly lower for patients who underwent CCTA versus usual care (HR: $0.81,95 \%$ CI $0.72-0.92$ ), while coronary revascularization rates were similar for the two groups (HR: $1.03,95 \% \mathrm{CI}$ : $0.87-1.21)$. The median length of hospitalization was higher for CCTA versus usual care ( 2.2 days vs. 2.0 days) as were the healthcare costs (\$9494 vs. \$8776).

\section{Clinical Implications}

The present study showed CCTA does not reduce the incidence of all-cause mortality and non-fatal cardiovascular outcomes among patients who present with ACS. However, CCTA potentially avoided the use of invasive coronary angiography as the trial demonstrates. However, this comes at the expense of increased length of hospital stay and associated healthcare costs.

A previous trial, ROMICAT-II, similarly looked at the use of CCTA versus usual care among patients presenting to the ED with chest pain [25]. Unlike RAPID-CCTA, the primary outcome was length of stay (LOS) and the trial showed that compared to usual care, CCTA decreased LOS by $7.6 \mathrm{~h}$ $(P<0.001)$, and more patients were discharged directly from the emergency department ( $47 \%$ vs. $12 \%, P<0.001)$. There were no undetected ACS events and no significant differences in major adverse cardiovascular events at 28 days. The use of CCTA did result in increased frequency downstream testing and higher radiation exposure. However, the cost of care was similar in both CCTA and usual care arms ( $\$ 4289$ and $\$ 4060$, respectively; $P=0.65$ ).

The null results of the RAPID-CCTA trial do not justify the routine use of CCTA. However, selective use of CCTA (or CAC) may have important health consequences that could ameliorate the excess of CVD in some patient populations. 
The utility of CCTA on reducing invasive coronary angiography is also an important outcome that may justify its use for avoiding invasive procedures. Further data on the sensitivity and specificity of CCTA in diagnosing obstructive CAD in the overall study population and subgroups is required to evaluate the use of CCTA for definitively ruling out obstructive disease.

\section{A Polypill for Primary Prevention of Cardiovascular Disease in Intermediate-Risk People}

The TIPS- 3 trial was a randomized clinical trial designed to study the safety and efficacy of a once-daily polypill (fixeddose combination of simvastatin, atenolol, ramipril, HCTZ) and aspirin versus placebo for reducing systolic blood pressure (SBP), LDL-C, and fatal or non-fatal cardiovascular events among intermediate-risk patients without a history of CVD [7].

\section{Study Overview}

The trial used a 2-by-2-by-2 factorial design whereby participants were assigned to receive a polypill $(40 \mathrm{mg}$ of simvastatin, $100 \mathrm{mg}$ of atenolol, $25 \mathrm{mg}$ of hydrochlorothiazide, and $10 \mathrm{mg}$ of ramipril) or placebo daily, aspirin $(75 \mathrm{mg}$ ) or placebo daily, and vitamin D or placebo daily. The following groups of patients were compared: (1) polypill alone versus placebo, (2) aspirin alone versus placebo, (3) polypill plus aspirin versus double placebo. The primary outcome for the polypill (alone or with aspirin) was a composite of cardiovascular death, myocardial infarction (MI), stroke, heart failure (HF), cardiac arrest, or revascularization. The primary outcome for the aspirin comparison was a composite of death from cardiovascular causes, myocardial infarction, or stroke. The trial also evaluated the safety of these drugs and adherence rates.

The trial included 5713 participants with mean age 64 years, $53 \%$ females, $37 \%$ with diabetes, and the mean duration of follow-up was 4.6 years. LDL-C was lowered by approximately $19 \mathrm{mg} / \mathrm{dL}$ and SBP was lowered by $5.8 \mathrm{mmHg}$ among those randomized to the polypill (with or without aspirin) compared to placebo. The primary outcome was non-significantly lower among those randomized to polypill versus placebo: $4.4 \%$ vs. $5.5 \%$ (HR: $0.79,95 \%$ CI: $0.63-1.00$ ) but significantly lower for combination polypill plus aspirin versus placebo: $4.1 \%$ vs. $5.8 \%$ (HR: $0.69,95 \%$ CI: $0.50-0.97)$. The primary outcome was nonsignificantly lower among those randomized to aspirin alone versus placebo: $4.1 \%$ vs. $4.7 \%$ (HR: $0.86,95 \%$ CI: $0.67-1.10)$ but the risk of stroke was significantly lower
(HR: $0.58,95 \%$ CI: $0.35,0.98)$. Adverse outcomes such as hypotension or dizziness were higher among those who received the polypill as compared to respective placebo groups. Muscle symptoms, gastrointestinal bleeding, dyspepsia, or gastritis were similar for the two groups. Importantly, major bleeding was reported in 9 participants in the polypill-plus aspirin group and 12 participants in the double-placebo group. Adherence rates were not ideal with $42 \%$ of study participants discontinuing the polypill by the end of the study.

\section{Clinical Implications}

The present trial demonstrated that the combination of a polypill (comprised of simvastatin and three blood-pressurelowering drugs) plus aspirin resulted in a $31 \%$ relative and $1.7 \%$ absolute risk reduction of adverse outcomes with the majority of benefit attributable to the polypill.

The reduction in cardiovascular risk factors in TIPS-3 and adherence rates were lower than expected which may have resulted in the smaller than expected 40 to $45 \%$ relative risk that was originally expected. However, the reduction in clinical outcomes was consistent with results from the HOPE-3 trial [26] which showed that the combination polypill of $10 \mathrm{mg}$ rosuvastatin statin plus two blood pressure-lowering drugs (16 mg candesartan plus $12.5 \mathrm{mg}$ hydrochlorothiazide) resulted in a $29 \%$ relative risk reduction over a median followup time of 5.6 years and the PolyIran trial [27] which showed that the combination of $81 \mathrm{mg}$ aspirin, $20 \mathrm{mg}$ atorvastatin, $20 \mathrm{mg}$ hydrochlorothiazide, and either $5 \mathrm{mg}$ enalapril or $40 \mathrm{mg}$ valsartan resulted in a $34 \%$ relative risk reduction over 5-year follow-up.

The polypill remains an attractive strategy for CVD prevention as it combines major drug classes (lipid-lowering, blood pressure-lowering, and aspirin) into one pill. In addition to targeting multiple risk factors simultaneously, the use of polypill likely improves adherence and minimize costs. However, adherence in the trial was low likely due to adverse side effects. As different drug classes have different side effects, it is important to address patient preferences and allergies when prescribing these drugs to maximize the possibility of adherence. A-One Pill to Treat Them All strategy may not yet be ready for prime time as both safety and efficacy need to be established for the majority of patients.

\section{A Three-Arm N-of-1 Trial with Statin, Placebo, and Tablet Free Periods, to Verify Side Effects and Identify Their Cause: the SAMSON Trial}

Statins are the cornerstone for the treatment of hypercholesterolemia and in the management of increased cardiovascular 
risk. However, statin-associated side effects, especially statinassociated muscle symptoms, are clinically important and have been reported in $10-25 \%$ of adults on statins $[28,29]$ and limit adherence to statin therapy [30]. The SAMSON study [8] was designed to assess the side effects associated with statin therapy and identify their cause.

\section{Study Overview}

In a three-arm N-of- 1 trial, 60 patients who stopped taking statin therapy due to intolerable side effects were included. At the start, patients were provided with four bottles each of atorvastatin $20 \mathrm{mg}$, placebo, and empty bottles. This continued for 12 months. The patients used a smartphone to register symptoms and were allowed to discontinue the tablet if the symptoms were intolerable. The primary endpoint was symptom intensity as assessed with the use of the nocebo ratio (i.e., the ratio of symptom intensity induced by taking placebo to the symptom intensity induced by taking a statin). Symptom scores ranged from 0 (no symptoms) to 100 (worst imaginable symptoms).

Mean symptom severity was substantially higher during the statin period $(16.3,95 \% \mathrm{CI}, 13.0$ to 19.6) compared to the no-tablet period $(8.0,95 \% \mathrm{CI}, 4.7$ to $11.3 P<0.001)$. However, mean symptom intensity was also substantially higher during the placebo period $(15.4,95 \% \mathrm{CI}, 12.1$ to 18.7) compared to the no treatment period $(\mathrm{P}<0.001)$ and there was no difference in symptom level between the statin period and the placebo period $(P=0.368)$. The study showed a pooled nocebo ratio of 0.90 . Importantly 6 months after completion of the trial, 30 of the patients $(50 \%)$ had successfully restarted statins, 4 planned to do so, and 1 could not be contacted. The remaining 25 patients were not receiving statins and were not planning to restart statins.

\section{Clinical Implications}

In patients who had discontinued statin therapy because of side effects, $90 \%$ of the symptom burden elicited by a statin challenge was also elicited by placebo. Half the trial patients were able to successfully restart statins. Patient-reported side effects to statin therapy are serious and should be taken seriously; however, some of the symptoms experienced due to statins are also experienced with placebo. Therefore, it is important to explore the etiology of symptoms experienced by patients and some patients may be able to restart statin therapy [31]. An important study limitation was that the trial did not have a wash-out period and only patients who developed symptoms within 2 weeks of starting statin therapy were recruited.

\section{Conclusion}

The clinical trials discussed above, which were presented at AHA Scientific Sessions 2020, represent the ongoing advancements made in the field of CVD prevention and highlight potential avenues for future research efforts. We highlight some promising novel therapies for the management of heart failure and cardiometabolic disease (such as diabetes and lipid disorders) and also provide new insights and possible solutions to routine challenges faced in the field of prevention.

\section{Compliance with Ethical Standards}

Conflict of Interest Salim S. Virani reports grants from Department of Veterans Affairs, World Heart Federation, Tahir and Jooma Family, and other from American College of Cardiology (Honorarium in my role as the Associate Editor for Innovations [acc.org]).

The other authors declare that they have no conflict of interest.

Human and Animal Rights and Informed Consent This article does not contain any studies with human or animal subjects performed by any of the authors.

\section{References}

Papers of particular interest, published recently, have been highlighted as:

- Of importance

•- Of major importance

1. Teerlink JR, Diaz R, Felker GM, McMurray JJV, Metra M, Solomon SD, et al. Cardiac myosin activation with Omecamtiv Mecarbil in systolic heart failure. N Engl J Med. 2020. https:// doi.org/10.1056/NEJMoa2025797 Randomized controlled trial showing that the primary outcome of cardiovascular death or heart failure was significantly reduced among patients with heart failure with reduced ejection fraction, New York Heart Association (NYHA) functional class II-IV and LVEF $\leq \mathbf{3 5 \%}$ receiving omecamtiv mecarbil compared to placebo.

2.• Bhatt DL, Szarek M, Steg PG, Cannon CP, Leiter LA, McGuire DK, et al. Sotagliflozin in Patients with Diabetes and Recent Worsening Heart Failure. N Engl J Med. 2020. https://doi.org/ 10.1056/NEJMoa2030183 In patients with a recent admission with $\mathrm{HF}$ and previous diagnosis of T2DM, sotagliflozin, when compared to placebo, significantly reduced rate of primary endpoint (cardiovascular mortality, HF hospitalizations).

3.• Nicholls SJ, Lincoff AM, Garcia M, Bash D, Ballantyne CM, Barter PJ, et al. Effect of High-Dose Omega-3 Fatty Acids vs Corn Oil on Major Adverse Cardiovascular Events in Patients at High Cardiovascular Risk: The STRENGTH Randomized Clinical Trial. JAMA. 2020. https://doi.org/10.1001/jama.2020. 22258 In a multi-centric, double-blinded, randomized, placebo-controlled trial of 13,078 patients with either established ASCVD, diabetes or or high-risk primary prevention patients on maximally tolerated statin therapy, use of of omega-3 CA failed to show significant reduction in cardiovascular events among patients with use compared with corn oil.

4. Kalstad AA, Myhre PL, Laake K, Tveit SH, Schmidt EB, Smith P, et al. Effects of n-3 fatty acid supplements in elderly patients after 
myocardial infarction. Circulation. 2020. https://doi.org/10.1161/ CIRCULATIONAHA.120.052209.

5. Rosenson RS, Burgess LJ, Ebenbichler CF, Baum SJ, Stroes ESG, Ali S, Khilla N, Hamlin R, Pordy R, Dong Y, Son V, Gaudet D. Evinacumab in Patients with Refractory Hypercholesterolemia. N Engl J Med. 2020 Dec 10;383(24):2307-19. https://doi.org/10. 1056/NEJMoa2031049.

6. Gray A. Early coronary CT angiography in patients with suspected or provisionally diagnosed acute coronary syndrome: the RAPID CTCA trial. Present Am Heart Assoc Sci Sessions. 2020; In 1,748 patients who presented to the emergency room with chest pain and prior history of coronary heart disease, troponin above the $99^{\text {th }}$ percentile, or an abnormal electrocardiogram, randomization to early coronary computed tomography angiography did not show reduction in primary outcome of non fatal myocardial infarction at one year compared to usual care.

7. Yusuf S, Joseph P, Dans A, Gao P, Teo K, Xavier D, et al. Polypill with or without aspirin in persons without cardiovascular disease. United States: N Engl J Med. 2020. https://doi.org/10.1056/ NEJMoa2028220.

8. Wood FA, Howard JP, Finegold JA, Nowbar AN, Thompson DM, Arnold AD, et al. N-of-1 Trial of a Statin, Placebo, or No Treatment to Assess Side Effects. N Engl J Med. 2020. https:// doi.org/10.1056/NEJMc2031173 A three arm N-of-1 clinical trial showed that among 60 patients who previously stopped taking statin therapy due to intolerable side-effects, who received statin, placebo and no-pill therapy, $90 \%$ of symptoms elicited by statin were also seen in placebo and at 6 months $50 \%$ of patients were able to re-start statin therapy.

9. Jia X, Al Rifai M, Hussain A, Martin S, Agarwala A, Virani SS. Highlights from studies in cardiovascular disease prevention presented at the Digital 2020 European Society of Cardiology Congress: Prevention Is Alive and Well. Curr Atheroscler Rep. 2020;22:72.

10. Jia X, Al Rifai M, Liu J, Agarwala A, Gulati M, Virani SS. Highlights of studies in cardiovascular disease prevention presented at the 2020 American College of Cardiology Annual Scientific Session. Curr Atheroscler Rep. 2020;22:32-6.

11. Al Rifai M, Jia X, Al-Mallah MH, Miedema MD, Martin SS, Virani SS. Major randomized clinical trials in cardiovascular disease prevention presented at the 2019 American College of Cardiology Annual Scientific Session. Curr Atheroscler Rep. 2019;21:31-3.

12. Jia X, Al Rifai M, Gluckman TJ, Birnbaum Y, Virani SS. Highlights from selected cardiovascular disease prevention studies presented at the 2019 European Society of Cardiology Congress. Curr Atheroscler Rep. 2019;21:46-7.

13. Teerlink JR, Felker GM, McMurray JJ, Solomon SD, Adams KF, Cleland JG, et al. Chronic Oral study of myosin activation to increase contractility in heart failure (COSMIC-HF): a phase 2, pharmacokinetic, randomised, placebo-controlled trial. Lancet. 2016;388:2895-903.

14. Packer M, Anker SD, Butler J, Filippatos G, Pocock SJ, Carson P, et al. Cardiovascular and renal outcomes with Empagliflozin in heart failure. N Engl J Med. 2020;383:1413-24.

15. McMurray JJV, Solomon SD, Inzucchi SE, Køber L, Kosiborod MN, Martinez FA, et al. Dapagliflozin in patients with heart failure and reduced ejection fraction. N Engl J Med. 2019;381:1995-2008.

16. Bhatt DL, Steg PG, Miller M, Brinton EA, Jacobson TA, Ketchum $\mathrm{SB}$, et al. Cardiovascular risk reduction with icosapent ethyl for hypertriglyceridemia. N Engl J Med. 2019;380:11-22.
17. Jia X, Koh S, Al Rifai M, Blumenthal RS, Virani SS. Spotlight on icosapent ethyl for cardiovascular risk reduction: evidence to date. Vasc Health Risk Manag. 2020;16:1-10.

18. ORIGIN Trial Investigators, Bosch J, Gerstein HC, Dagenais GR, Díaz R, Dyal L, et al. N-3 fatty acids and cardiovascular outcomes in patients with dysglycemia. N Engl J Med. 2012;367(4):309-18.

19. Aung T, Halsey J, Kromhout D, Gerstein HC, Marchioli R, Tavazzi L, et al. Associations of Omega-3 fatty acid supplement use with cardiovascular disease risks: meta-analysis of 10 trials involving 77 917 individuals. JAMA Cardiol. 2018;3(3):225-34.

20. Yokoyama M, Origasa H, Matsuzaki M, Matsuzawa Y, Saito Y, Ishikawa $\mathrm{Y}$, et al. Effects of eicosapentaenoic acid on major coronary events in hypercholesterolaemic patients (JELIS): a randomised open-label, blinded endpoint analysis. Lancet. 2007;369(9567):1090-8.

21. Hussain A, Ballantyne CM, Saeed A, Virani SS. Triglycerides and ASCVD risk reduction: recent insights and future directions. Curr Atheroscler Rep. 2020;22(7):25-8.

22. Khera AV, Won HH, Peloso GM, Lawson KS, Bartz TM, Deng X, et al. Diagnostic yield and clinical utility of sequencing familial hypercholesterolemia genes in patients with severe hypercholesterolemia. J Am Coll Cardiol. 2016;67:2578-89.

23. Dewey FE, Gusarova V, Dunbar RL, O'Dushlaine C, Schurmann $\mathrm{C}$, Gottesman O, et al. Genetic and pharmacologic inactivation of ANGPTL3 and cardiovascular disease. N Engl J Med. 2017;377(3): 211-21.

24. Jia X, Liu J, Mehta A, Ballantyne CM, Virani SS. Lipid-lowering biotechnological drugs: from monoclonal antibodies to antisense therapies-a clinical perspective. Cardiovasc Drugs Ther. 2020. https://doi.org/10.1007/s10557-020-07082-x.

25. Hoffmann U, Truong QA, Schoenfeld DA, Chou ET, Woodard PK, et al. Coronary CT angiography versus standard evaluation in acute chest pain. N Engl J Med. 2012;367(4):299-308.

26. Yusuf S, Bosch J, Dagenais G, Zhu J, Xavier D, Liu L, et al. Cholesterol lowering in intermediate-risk persons without cardiovascular disease. Cholesterol lowering in intermediate-risk persons without cardiovascular disease. N Engl J Med. 2016;374:2021-31.

27. Roshandel G, Khoshnia M, Poustchi H, Hemming K, Kamangar F, Gharavi A, et al. Effectiveness of polypill for primary and secondary prevention of cardiovascular diseases (PolyIran): a pragmatic, cluster-randomised trial. Lancet. 2019;394:672-83.

28. Bruckert E, Hayem G, Dejager S, Yau C, Bégaud B. Mild to moderate muscular symptoms with high-dosage statin therapy in hyperlipidemic patients-the PRIMO study. Cardiovasc Drugs Ther. 2005; 19:403-14.

29. Cohen JD, Brinton EA, Ito MK, Jacobson TA. Understanding statin use in America and gaps in patient education (USAGE): an internetbased survey of 10,138 current and former statin users. J Clin Lipidol. 2012;6:208-15.

30. Virani SS, Akeroyd JM, Ahmed ST, Krittanawong C, Martin LA, Slagle J, et al. The use of structured data elements to identify ASCVD patients with statin-associated side effects: Insights from the Department of Veterans Affairs. J Clin Lipidol. 2019;3:797803.e1.

31. Ahmed ST, Akeroyd JM, Mahtta D, Street R, Slagle J, Navar AM, et al. Shared decisions: a qualitative study on clinician and patient perspectives on statin therapy and statin-associated side effects. J Am Heart Assoc. 2020;9:e017915.

Publisher's Note Springer Nature remains neutral with regard to jurisdictional claims in published maps and institutional affiliations. 